\title{
The Effect of Treatment on Survival in Patients with Advanced Laryngeal Carcinoma
}

Christine G. Gourin, MD; Bryant T. Conger, BS; W. Chris Sheils, MD; Paul A. Bilodeau, MD; Teresa A. Coleman, MD; Edward S. Porubsky, MD

Objectives/Hypothesis: Over the last 2 decades, survival from laryngeal cancer has decreased. We sought to identify factors associated with decreased survival in laryngeal cancer.

Methods: Patients diagnosed with laryngeal squamous cell carcinoma from 1985 to 2002 were retrospectively reviewed.

Results: A total of 451 patients met study criteria. Five-year survival rates were $85 \%$ for stage I, $77 \%$ for stage II, $51 \%$ for stage III, and $35 \%$ for stage IV disease. Survival for patients with stage I-III disease was similar for patients treated operatively or nonoperatively $(P=.4)$. However, patients with stage III disease treated nonoperatively had worse survival with radiation alone (XRT) compared to chemoradiation $(\mathrm{CR})(P=.006)$. Patients with stage IV disease had significantly better survival with surgery $(49 \%)$ than CR $(21 \%)$ or XRT alone $(14 \%)(P<.0001)$. Analysis by primary tumor stage demonstrated that survival for T1-T3 disease was independent of treatment modality $(P=.2)$; however, for T4 patients, operative treatment was associated with significantly better survival $(55 \%)$ than CR $(25 \%)$ or XRT $(0 \%)(P<$ .0001). Proportional hazards models confirmed significantly worse survival for stage IV, T4, N2 or N3 disease, and nonoperative treatment. For T4 disease, after controlling for nodal status, nonoperative treatment was the only significant predictor of worse survival.

From the Department of Otolaryngology-Head and Neck Surgery (c.G.G.), Johns Hopkins University, Baltimore, Maryland, Department of Otolaryngology-Head and Neck Surgery (B.T.C., E.S.P.), Department of Radiology (w.c.s.), and Department of Medicine (P.A.B., T.A.c.), Medical College of Georgia, Augusta, Georgia, U.S.A.

Editor's Note: This Manuscript was accepted for publication March 12, 2009.

Presented at the 7th International Meeting of the American Head and Neck Society, San Francisco, California, U.S.A., July 21, 2008

Send correspondence to Christine G. Gourin, MD, FACS, Department of Otolaryngology-Head and Neck Surgery, Johns Hopkins University, 601 N. Caroline Street, Suite 6260, Baltimore, MD 21287. E-mail: cgourin1@jhmi.edu

DOI: 10.1002/lary.20477
Conclusions: Primary surgical treatment is associated with improved survival for patients with stage IV disease and specifically T4 primary tumors. These data suggest that the observed national decrease in survival from laryngeal cancer may be due to a shift toward nonoperative treatment in that subset of patients with advanced primary disease.

Key Words: Larynx, squamous cell cancer, head and neck neoplasms, chemoradiation, surgery, radiation.

Laryngoscope, 119:1312-1317, 2009

\section{INTRODUCTION}

In the past 2 decades, there has been a paradigm shift in the treatment of advanced laryngeal cancer, with increased utilization of chemoradiation (CR) and decreased use of primary surgical treatment. ${ }^{1-3}$ Interest in the use of induction chemotherapy in head and neck cancer treatment was validated by the publication of a landmark study conducted by the Veterans Affairs (VA) Laryngeal Cancer Study Group in $1991 .{ }^{4}$ This prospective, randomized controlled clinical trial showed that organ preservation in patients with advanced laryngeal cancer with $\mathrm{CR}$ could be attempted without compromising survival. Increasingly, the use of induction chemotherapy utilized in the VA study has given way to concurrent CR regimens based on the results of the Radiation Therapy Oncology Group (RTOG) Trial 91-11 showing improved local control rates. ${ }^{5,6}$ These clinical trials have impacted practice patterns, as reflected in the National Cancer Data Base (NCDB) by an increase in the use of $\mathrm{CR}$ in both community hospitals and academic centers, ${ }^{3}$ and a corresponding decrease in the use of total laryngectomy. ${ }^{2}$

During this same time period, survival among patients with laryngeal cancer recorded in the NCDB has decreased. ${ }^{2}$ This increase in mortality parallels the observed trend of increasing use of nonoperative treatment in these patients and inappropriate use of nonoperative treatment, and was not due to an increased incidence of advanced stage disease. ${ }^{2}$ In 
contrast to the results of the VA study and RTOG 91-11, an analysis of NCDB laryngeal cancer patients found an increased risk of death for stage IV patients treated with $\mathrm{CR},{ }^{7}$ suggesting there may be differences in patient populations and the application of treatment between the clinical trial setting and the larger community. We reviewed our institutional experience to identify factors associated with decreased survival in laryngeal cancer.

\section{MATERIALS AND METHODS}

The medical records of all patients evaluated for laryngeal squamous cell cancer at the Medical College of Georgia from 1985 to 2002 were reviewed in compliance with the Health Insurance Portability and Accountability Act. Patients with a history of prior treatment for head and neck cancer, known distant metastatic disease, nonsquamous neoplasms, and who did not complete treatment and without follow-up were excluded.

Primary surgical therapy included cordectomy, partial, total, or extended total laryngectomy. Elective neck dissection was performed for clinically node negative patients at risk for nodal metastases with removal of levels II to IV on the ipsilateral side for advanced laryngeal tumors and bilateral elective neck dissection for supraglottic primary tumors. Modified radical neck dissection was performed for clinically positive nodal disease. Postoperative radiation (XRT) was administered for three or more pathologically involved nodes, involvement of multiple levels, extracapsular spread, and adverse pathologic primary site features including T4 disease, perineural invasion, or lymphovascular invasion. Primary XRT alone was used as a nonoperative treatment for stage I or II disease. Chemoradiation, consisting of cisplatin with or without 5-fluorouracil, taxol, or primary XRT alone were used as primary nonoperative therapy for advanced stage disease (III/IV). Salvage laryngectomy was performed for patients without a complete response to XRT or CR or who developed recurrence. Patients with persistent nodal disease following XRT or CR or with initial stage N2 or N3 disease treated with CR underwent post-treatment modified radical neck dissection.

Standard statistical analysis, including chi-square analysis, Kaplan-Meier survival curves, and proportional hazards models was performed using Prism 5.0 (GraphPad Software, San Diego, CA) and Stata 10 (StataCorp, College Station, TX). Significance was attributed to a $P$ value $\leq .05$. This protocol was reviewed and approved by the Medical College of Georgia institutional review board.

\section{RESULTS}

A total of 451 patients met inclusion criteria with a mean follow-up time of 35 months (Table I). The majority of patients were white $(58 \%)$, male $(83 \%)$, and smokers (90\%). The mean age was 56 years (range, 3287 years). The supraglottic larynx represented the most common primary site $(50 \%)$, followed by the glottic larynx (30\%), and transglottic tumors in whom the specific laryngeal site of origin could not be determined (20\%). Advanced primary site disease (T3 or T4) was present in the majority of patients $(59 \%)$ as was advanced (III/IV) TNM stage $(69 \%)$. Age, sex, race, smoking history, and comorbidity grade did not differ significantly between patients treated operatively or nonoperatively. Surgery was used as the primary therapy in 259 (57\%) patients, XRT only was used in $124(27 \%)$ patients, and CR was used in $68(16 \%)$ patients.
Significant differences existed for primary site and stage between patients by treatment. Patients with transglottic tumors were more often treated surgically (72\%). A significantly greater number of patients with T2 $(63 \%)$ or stage II $(75 \%)$ disease were treated nonoperatively, whereas patients with T3 $(73 \%)$ or stage III $(71 \%)$ disease were more often treated surgically. The majority of patients with advanced $\mathrm{T}$ stage $(67 \%)$ or TNM stage $(64 \%)$ disease were treated surgically $(P<$ .0001).

Five-year survival rates were $85 \%$ for stage I, $77 \%$ for stage II, $51 \%$ for stage III, and $35 \%$ for stage IV disease (Fig. 1). Survival rates for stage I-III disease did not differ significantly between patients treated operatively or nonoperatively $(P=.4)$. However, patients with stage III disease treated nonoperatively had worse 5year survival with XRT alone (22\%) compared to CR $(57 \%)(P=.006)$. Patients with stage IV disease had significantly better survival with operative treatment $(49 \%)$ than CR $(21 \%)$ or XRT alone $(14 \%)(P<.0001)$ (Fig. 2$)$. Analysis by primary tumor stage demonstrated that survival for $\mathrm{T} 1$ to $\mathrm{T} 3$ disease was independent of treatment modality $(P=.2)$; however, in T4 patients, after controlling for clinical node stage and inoperable (T4b) disease, operative treatment was associated with significantly better survival $(55 \%)$ than $\mathrm{CR}(25 \%)$ or XRT $(0 \%)(P<$ .0001) (Fig. 3).

Cox proportional hazards models were used to analyze the effects of multiple simultaneous risk factors that could influence disease-specific survival (Table II). Because only eight patients had no history of tobacco use, smoking status was not entered into the model. Stage IV disease, T4 tumor stage, N3 nodal status, and nonoperative treatment were associated with significantly decreased disease-specific survival times. Models were sequentially built to further examine patients with stage IV disease to identify variables that were significantly associated with disease-specific survival. The resulting proportional hazards model of patients with stage IV disease included tumor stage, nodal status, and treatment. Disease-specific survival was significantly worse for patients with T4 tumors (hazard ratio [HR] 2.0), N2 (HR 2.3) and N3 (HR 3.0) nodal status, and nonoperative treatment with CR (HR 2.1) and XRT (HR 3.5) (Table III). Proportional hazards analysis of T4 patients showed that after controlling for nodal status, treatment was significantly associated with disease-specific survival, with an increased hazard ratio or odds of death for patients treated with CR (HR 2.0) or XRT alone (HR 7.2) compared to total laryngectomy followed by postoperative XRT (Table IV).

\section{DISCUSSION}

The results of this analysis demonstrate that nonoperative treatment is associated with poorer survival for patients with stage IV laryngeal cancer, and in particular for that subset of patients with T4 disease. Although nodal status was associated with an increased risk of disease-specific mortality in stage IV disease, only treatment was significant in the analysis of $\mathrm{T} 4$ 


\begin{tabular}{|c|c|c|c|c|}
\hline \multicolumn{5}{|c|}{$\begin{array}{c}\text { TABLE I. } \\
\text { Population Characteristics. }\end{array}$} \\
\hline Patient Variable & Total $(\%)(n=451)$ & Operative (\%) $(n=259)$ & Nonoperative (\%) $(n=192)$ & $P$ Value \\
\hline Age (yrs) & & & & .8674 \\
\hline$\leq 50$ & $125(27.7 \%)$ & $71(27.4 \%)$ & $54(28.1 \%)$ & \\
\hline$>50$ & $326(72.3 \%)$ & $188(72.6 \%)$ & $138(71.9 \%)$ & \\
\hline Sex & & & & .2679 \\
\hline Male & $375(83.1 \%)$ & $211(81.5 \%)$ & $164(85.4 \%)$ & \\
\hline Female & $76(16.9 \%)$ & $48(18.5 \%)$ & $28(14.6 \%)$ & \\
\hline Race & & & & .8300 \\
\hline Black & $190(42.1 \%)$ & $108(41.7 \%)$ & $82(42.7 \%)$ & \\
\hline White & 261 (57.9\%) & $151(58.3 \%)$ & $110(57.3 \%)$ & \\
\hline Smoking history & & & & .5219 \\
\hline None & $8(1.8 \%)$ & $5(1.9 \%)$ & $3(1.6 \%)$ & \\
\hline Past or present & $405(89.8 \%)$ & $229(88.4 \%)$ & $176(91.7 \%)$ & \\
\hline Not known & $38(8.4 \%)$ & $25(9.7 \%)$ & $13(6.7 \%)$ & \\
\hline T stage & & & & $<.0001$ \\
\hline $\mathrm{T} 1$ & $89(19.7 \%)$ & $46(17.8 \%)$ & $43(22.4 \%)$ & \\
\hline $\mathrm{T} 2$ & $98(21.7 \%)$ & $36(13.9 \%)$ & $62(32.3 \%)$ & \\
\hline T3 & 137 (30.4\%) & $100(38.6 \%)$ & 37 (19.3\%) & \\
\hline $\mathrm{T} 4$ & $127(28.2 \%)$ & $77(29.7 \%)$ & $50(26.0 \%)$ & \\
\hline $\mathrm{N}$ stage & & & & .0055 \\
\hline No & $258(57.2 \%)$ & $149(57.5 \%)$ & $109(56.8 \%)$ & \\
\hline $\mathrm{N} 1$ & $63(14.0 \%)$ & $45(17.4 \%)$ & $18(9.4 \%)$ & \\
\hline $\mathrm{N} 2$ & $102(22.6 \%)$ & $56(21.6 \%)$ & $46(23.9 \%)$ & \\
\hline N3 & $28(6.2 \%)$ & $9(3.5 \%)$ & 19 (9.9\%) & \\
\hline TNM stage & & & & $<.0001$ \\
\hline I & $80(17.7 \%)$ & 45 (17.4\%) & 35 (18.2\%) & \\
\hline II & $60(13.3 \%)$ & 15 (5.8\%) & 45 (23.4\%) & \\
\hline III & 116 (25.7\%) & $82(31.6 \%)$ & 34 (17.7\%) & \\
\hline IV & 195 (43.3\%) & 117 (45.2\%) & 78 (40.6\%) & \\
\hline Primary site & & & & .003 \\
\hline Glottis & 134 (29.7\%) & $66(25.5 \%)$ & 68 (35.4\%) & \\
\hline Supraglottis & 225 (49.9\%) & $128(49.4 \%)$ & 97 (50.5\%) & \\
\hline Subglottis & $3(0.7 \%)$ & $1(0.4 \%)$ & $2(1.0 \%)$ & \\
\hline Transglottic & 89 (19.7\%) & $64(24.7 \%)$ & 25 (13.0\%) & \\
\hline Comorbidity & & & & .4219 \\
\hline Low grade & 297 (65.9\%) & 175 (67.6\%) & $122(63.5 \%)$ & \\
\hline High grade & 154 (34.1\%) & 84 (32.4\%) & 70 (36.5\%) & \\
\hline
\end{tabular}

patients, suggesting that T4 status may the single most important predictor of survival differences between surgical and nonoperative treatment for patients with stage IV laryngeal cancer.

The VA study included patients with stage IV disease and demonstrated that salvage laryngectomy was required significantly more often for patients with stage IV and T4 cancers. ${ }^{4,8}$ Closer inspection of the VA study data reveals that a subset of 41 patients with T4N0 disease had reduced survival in the CR arm compared to the surgical $\operatorname{arm}(P=.0098$, log-rank test $)$. As a result, patients with large volume T4 disease, defined as extension through cartilage or greater than $1 \mathrm{~cm}$ of base of tongue involvement were excluded from the RTOG 91-11 trial, but T4 tumors with less extensive involvement were included. ${ }^{5}$ The RTOG 91-11 trial demonstrated significantly poorer survival for patients who required salvage laryngectomy compared to those who did not $(P$ $=.018$, log-rank test). ${ }^{6}$ It is not clear if differences in disease-free survival and laryngectomy-free survival existed between patients of different primary tumor stage after controlling for nodal status.

It is unclear whether the survival results from these randomized clinical trials translate outside of the clinical trial setting. One factor associated with decreased survival rates in the NCDB was an increase in the use of nonoperative treatment, and in particular, XRT alone in advanced stage disease compared to earlier years. $^{2}$ Selection criteria and treatment protocols for both the VA laryngeal study and RTOG 91-11 were 


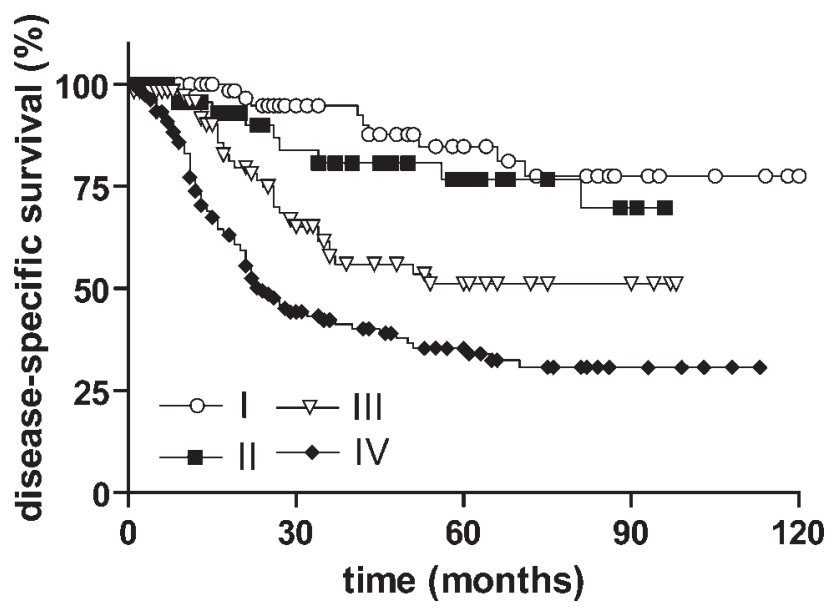

Fig. 1. Disease-specific survival by TNM stage.

strict, and patients were managed by an experienced, multidisciplinary team with active participation by head and neck surgeons. Patients with T4 disease and N2 or N3 nodal status require special evaluation before treatment to determine appropriateness of nonsurgical therapy, and close monitoring after treatment for salvage of patients with less than a complete response. ${ }^{6,9,10}$ In contrast, analysis of Commission on Cancer data obtained from multiple hospitals shows overall diversity in laryngeal cancer management by site and stage. ${ }^{11}$

T4 lesions are defined as tumors that invade through the thyroid cartilage and/or invade tissues beyond the larynx. ${ }^{12}$ Patients with T4 lesions comprised $26 \%$ of participants in the VA study, with T4N0 disease present in $12 \%$ of patients. Patients with low-volume T4 disease were incorporated into RTOG 91-11 and represented $10 \%$ or less of each study arm. T4 disease thus represents a minority of patients in organ preservation trials, and the American Society of Clinical Oncology's clinical practice guidelines for the use of larynx-preservation strategies in laryngeal cancer recommend that T4

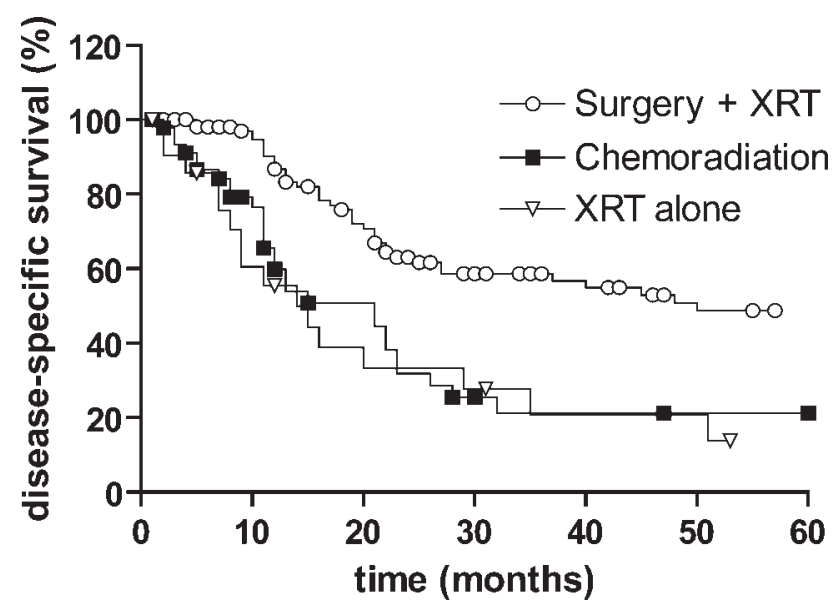

Fig. 2. Disease-specific survival by treatment for patients with stage IV larynx cancer. Operative treatment was associated with significantly better survival than nonoperative treatment $(P<$ .0001). XRT $=$ radiation therapy.

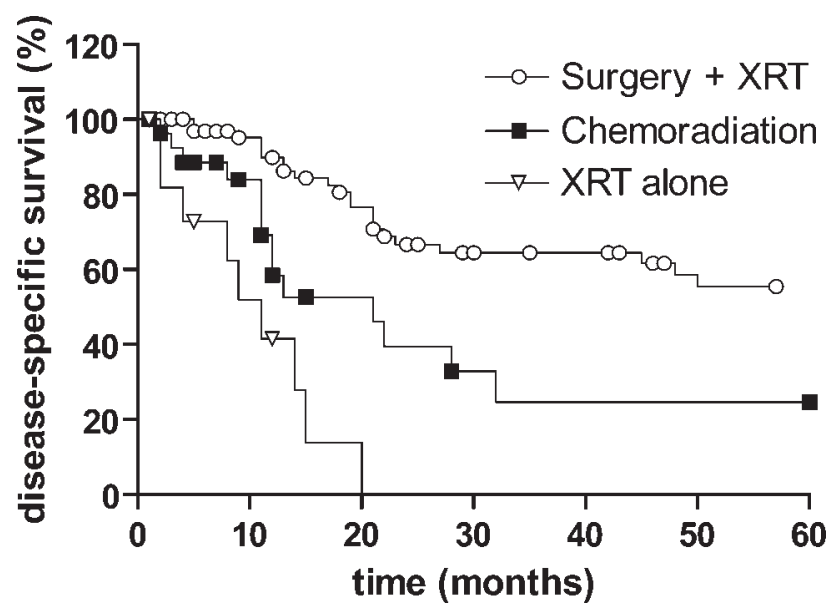

Fig. 3. Disease-specific survival by treatment for patients with T4 larynx cancer. Operative treatment was associated with significantly better survival than nonoperative treatment $(P<.0001)$. $\mathrm{XRT}=$ radiation therapy.

TABLE II.

Cox Proportional Hazards Analysis of the Effect of Variables on Survival Among Patients With Laryngeal Cancer $(n=451)$.

\begin{tabular}{|c|c|c|c|c|}
\hline & $\begin{array}{l}\text { Hazard } \\
\text { Ratio }\end{array}$ & $\begin{array}{l}\text { Standard } \\
\text { Error }\end{array}$ & $95 \% \mathrm{Cl}$ & $P$ Value \\
\hline \multicolumn{5}{|l|}{ Age (yrs) } \\
\hline$\leq 50$ & 1.000 & & & \\
\hline$>50$ & 1.288 & 0.306 & $0.808-2.052$ & .287 \\
\hline \multicolumn{5}{|l|}{ Sex } \\
\hline Male & 1.000 & & & \\
\hline Female & 0.666 & 0.183 & $0.388-1.143$ & .141 \\
\hline \multicolumn{5}{|l|}{ Race } \\
\hline White & 1.000 & & & \\
\hline Black & 1.441 & 0.294 & $0.966-2.149$ & .073 \\
\hline \multicolumn{5}{|l|}{ TNM stage } \\
\hline Stage I & 1.000 & & & \\
\hline Stage II & 2.238 & 1.249 & $0.850-6.662$ & .099 \\
\hline Stage III & 2.717 & 2.218 & $0.549-13.455$ & .220 \\
\hline Stage IV & 3.427 & 1.996 & $1.094-10.737$ & .034 \\
\hline \multicolumn{5}{|l|}{ Tumor stage } \\
\hline $\mathrm{T} 1$ & 1.000 & & & \\
\hline T2 & 0.471 & 0.323 & $0.123-1.808$ & .273 \\
\hline T3 & 1.683 & 0.630 & $0.808-3.505$ & .164 \\
\hline $\mathrm{T} 4$ & 1.962 & 0.683 & $0.992-3.881$ & .053 \\
\hline \multicolumn{5}{|l|}{ Nodal status } \\
\hline No & 1.000 & & & \\
\hline N1 & 1.473 & 0.454 & $0.804-2.698$ & .209 \\
\hline N2 & 1.846 & 0.591 & $0.985-3.459$ & .055 \\
\hline N3 & 2.258 & 0.928 & $1.001-5.056$ & .048 \\
\hline \multicolumn{5}{|l|}{ Treatment } \\
\hline Surgery & 1.000 & & & \\
\hline XRT & 2.928 & 0.726 & $1.800-4.760$ & $<.001$ \\
\hline $\mathrm{CR}$ & 2.395 & 0.589 & $1.479-3.880$ & $<.001$ \\
\hline \multicolumn{5}{|l|}{ Comorbidity } \\
\hline Low grade & 1.000 & & & \\
\hline High grade & 1.063 & 0.721 & $0.721-1.566$ & .757 \\
\hline
\end{tabular}

$\mathrm{Cl}=$ confidence interval. 


\begin{tabular}{|c|c|c|c|c|}
\hline \multicolumn{5}{|c|}{$\begin{array}{c}\text { TABLE III. } \\
\text { Cox Proportional Hazards Analysis of the Effect of } \\
\text { Variables on Survival Among Patients With Stage IV } \\
\text { Laryngeal Cancer ( } n=195) \text {. }\end{array}$} \\
\hline & Hazard Ratio & Standard Error & $95 \% \mathrm{Cl}$ & $P$ Value \\
\hline \multicolumn{5}{|c|}{ Tumor stage } \\
\hline $\mathrm{T} 1$ & 1.000 & & & \\
\hline $\mathrm{T} 2$ & 0.412 & 0.282 & $0.107-1.573$ & .195 \\
\hline T3 & 1.938 & 0.678 & $0.976-3.848$ & .058 \\
\hline $\mathrm{T} 4$ & 2.029 & 0.712 & $1.020-4.036$ & .044 \\
\hline \multicolumn{5}{|c|}{ Nodal status } \\
\hline No & 1.000 & & & \\
\hline N1 & 1.430 & 0.443 & $0.778-2.628$ & .249 \\
\hline N2 & 2.292 & 0.537 & $1.448-3.627$ & $<.001$ \\
\hline N3 & 2.960 & 1.059 & $1.467-5.972$ & .002 \\
\hline \multicolumn{5}{|l|}{ Treatment } \\
\hline Surgery & 1.000 & & & \\
\hline XRT & 3.521 & 0.890 & $2.145-5.780$ & $<.001$ \\
\hline $\mathrm{CR}$ & 2.138 & 0.539 & $1.305-3.505$ & .003 \\
\hline
\end{tabular}

$\mathrm{Cl}=$ confidence interval; $\mathrm{CR}=$ chemoradiation; $\mathrm{XRT}=$ radiation

lesions with tumor invasion through cartilage are poor candidates for laryngeal preservation and should undergo total laryngectomy. ${ }^{12}$ However, T4 cancers are often combined with T3 disease under the heading of advanced primary site disease ${ }^{12}$ or with other stages under the umbrella of advanced stage cancer ${ }^{4,5}$ in result reporting, which may encourage broad application of CR unless particular emphasis is placed on the poorer outcomes with CR for advanced T4 lesions.

Furthermore, the accuracy of preoperative imaging in determining laryngeal cartilage invasion varies from $33 \%$ to $75 \%$, and soft tissue extension of tumors may not be distinguished easily from inflammatory changes and edema, particularly in the absence of overt cartilage destruction. ${ }^{13}$ This can lead to inappropriate use of nonoperative therapy for inadequately staged disease. Neoplastic invasion of the thyroid cartilage is more likely in advanced glottic cancers because of the proximity of Broyle's ligament to the thyroid cartilage, and is associated with limited radiocurability. T4 tumors without cartilage destruction that involve the oropharynx or hypopharynx may take on the survival characteristics of primary oropharyngeal and hypopharyngeal tumors. Tumors with oropharyngeal involvement may be amenable to CR, but organ preservation strategies for hypopharyngeal cancer are associated with a lower laryngeal preservation rate ${ }^{14}$ and poorer survival when compared to primary surgical therapy followed by $\mathrm{CR}^{15}$ It is not possible to determine from this retrospective review if $\mathrm{T} 4$ cancer in this series was predominantly associated with cartilage invasion, extralaryngeal spread, or low volume T4 disease. However, our data suggests that primary surgical therapy results in improved survival for patients with $\mathrm{T} 4$ disease.

Advanced stage nodal disease is a known adverse prognostic factor and is related to the poorer survival of stage IV disease, which includes N2 and N3 nodal dis- ease by definition. When organ preservation therapy is used in patients with advanced neck disease, the response in the neck may be independent of the response at the primary site. Follow-up of patients in the VA study showed poorer survival in patients with N2/N3 disease and a partial response in the neck who subsequently required salvage neck dissection because of inability to control disease in the neck, compared to those with a complete response. ${ }^{9}$ The incorporation of early, planned post-treatment neck dissection in such patients resulted in improved regional control and no difference in survival between patients with a partial response compared to complete responders. ${ }^{10}$ As a result, post-treatment neck dissection is recommended for patients with $\mathrm{N} 2$ or N3 disease with residual nodal disease following CR. ${ }^{16,17}$ Because not all residual nodes harbor viable tumor, positron emission tomographycomputed tomography (PET-CT) is being investigated as a method of identifying those patients who would benefit from neck dissection, but is controversial in patients with residual adenopathy. Clearly, however, there remains a role for surgery in patients with advanced nodal disease.

Any paradigm for organ preservation in advanced laryngeal cancer should be expected to result in equivalent survival rates when compared to the gold standard of laryngectomy with postoperative XRT. The enthusiasm for laryngeal preservation, given the perceived mutilation of total laryngectomy, may result in a tendency to generalize the results of clinical trial data and apply these beyond the limits set by clinical trial data. Organ preservation should not be used as a method of selecting patients for salvage surgery, but should only be considered when survival and function can be anticipated to be equivalent to the standard of laryngectomy with postoperative XRT, and when such an approach can be delivered by a multidisciplinary treatment team with the necessary expertise. In addition to poorer survival with the use of $\mathrm{CR}$ for $\mathrm{T} 4$ disease, the incidence of severe late toxicity related to the use of $\mathrm{CR}$, including laryngeal dysfunction and prolonged feeding tube dependence, is significantly higher for advanced primary stage disease. ${ }^{18}$ Patient wishes and clinician desires

TABLE IV.

Cox Proportional Hazards Analysis of the Effect of Variables on Survival Among Patients With T4 Laryngeal Cancer $(n=127)$.

\begin{tabular}{llccr}
\hline & $\begin{array}{c}\text { Hazard } \\
\text { Ratio }\end{array}$ & $\begin{array}{c}\text { Standard } \\
\text { Error }\end{array}$ & $95 \% \mathrm{Cl}$ & $P$ Value \\
\hline Nodal status & & & & \\
N0 & 1.000 & & & \\
N1 & 0.947 & 0.502 & $0.335-2.676$ & .918 \\
N2 & 1.695 & 0.586 & $0.861-3.340$ & .127 \\
N3 & 1.684 & 0.919 & $0.577-4.912$ & .340 \\
Treatment & & & & \\
Surgery & 1.000 & & & \\
XRT & 7.172 & 0.322 & $3.140-16.380$ & $<.001$ \\
CR & 2.04 & 0.728 & $1.016-4.111$ & .045 \\
\hline \hline
\end{tabular}

$\mathrm{Cl}=$ confidence interval; $\mathrm{CR}=$ chemoradiation; $\mathrm{XRT}=$ radiation . 
often factor into the decision to pursue nonoperative treatment: however, it is incumbent upon us to adequately counsel patients about the risks and assist them in making informed decisions.

\section{CONCLUSION}

The results of this analysis demonstrate that primary surgical treatment is associated with improved survival for patients with stage IV laryngeal cancer and specifically T4 primary tumors. Although nodal status was associated with an increased risk of disease-specific mortality in stage IV disease, only treatment was significant in the analysis of T4 patients, suggesting that T4 status may be the single most important predictor of survival differences between surgical and nonoperative treatment for patients with stage IV disease. These data suggest that the observed national decrease in survival from laryngeal cancer may be due to a shift toward nonoperative treatment in that subset of patients with advanced primary disease.

\section{BIBLIOGRAPHY}

1. Hoffman HT, Karnell LH, Funk GF, et al. The National Cancer Data Base report on cancer of the head and neck. Arch Otolaryngol Head Neck Surg 1998;124:951-962.

2. Hoffman HT, Porter K, Karnell LH, et al. Laryngeal cancer in the United States: changes in demographics, patterns of care, and survival. Laryngoscope 2006;116(suppl 111): $1-13$.

3. Chen AY, Schrag N, Hao Y, et al. Changes in treatment of advanced laryngeal cancer 1985-2001. Otolaryngol Head Neck Surg 2006;135:831-837.

4. The Department of Veterans Affairs Laryngeal Cancer Study Group. Induction chemotherapy plus radiation compared with surgery plus radiation in patients with advanced laryngeal cancer. $N$ Eng J Med 1991;324: $1685-1690$

5. Forastiere A, Goepfert H, Maor M, et al. Concurrent chemotherapy and radiotherapy for organ preservation in advanced laryngeal cancer. $N$ Eng $J$ Med 2003;349: 2091-2098.
6. Weber RS, Berkey BA, Forastiere A, et al. Outcome of salvage total laryngectomy following organ preservation therapy: the Radiation Therapy Oncology Group Trial 91-11. Arch Otolaryngol Head Neck Surg 2003;129: $44-49$.

7. Chen AY, Halpern H. Factors predictive of survival in advanced laryngeal cancer. Arch Otolaryngol Head Neck Surg 2007;133:1270-1276.

8. Forastiere AA. Larynx preservation trials: a critical appraisal. Sem Rad Oncol 1998;8:254-261.

9. Wolf GT, Fisher SG. Effectiveness of salvage neck dissection for advanced regional metastases when induction chemotherapy and radiation are used for organ preservation. Laryngoscope 1992;102:934-939.

10. Thomas GR, Greenberg J, Wu KT, et al. Planned early neck dissection before radiation for persistent neck nodes after induction chemotherapy. Laryngoscope 1997;107: 1129-1137.

11. Shah JP, Karnell LH, Hoffman HT, et al. Patterns of care for cancer of the larynx in the United States. Arch Otolaryngol Head Neck Surg 1997;123:475-483.

12. Pfister DG, Laurie SA, Weinstein GS, et al. American Society of Clinical Oncology clinical practice guidelines for the use of larynx-preservation strategies in the treatment of laryngeal cancer. J Clin Oncol 2006;24:3693-3704.

13. Ampil FL, Nathan CA, Caldito G, et al. Total laryngectomy and postoperative radiation for T4 laryngeal cancer: a 14year review. Am J Otolaryngol 2004;25:88-93.

14. Lefebvre JL, Chevalier D, Luboinski B, et al. Larynx preservation in piriform sinus cancer: preliminary results of a European Organization for Research and Treatment of Cancer phase III trial. J Natl Cancer Inst 1996;88: 890-899.

15. Tsou YA, Lin MH, Hua CH, et al. Survival outcome by early chemoradiation therapy salvage or early surgical salvage for the treatment of hypopharyngeal cancer. Otolaryngol Head Neck Surg 2007;137:711-716.

16. Vedrine PO, Thariat J, Hitier M, et al. Need for neck dissection after radiochemotherapy? A study of the French GETTEC Group. Laryngoscope 2008;118:1775-1780.

17. Brizel DM, Prosnitz RG, Hunter S, et al. Necessity for adjuvant neck dissection in setting of concurrent chemoradiation for advanced head and neck cancer. Int $J$ Radiat Oncol Biol Phys 2004;58:1418-1423.

18. Machtay M, Moughan J, Trotti A, et al. Factors associated with severe late toxicity after concurrent chemoradiation for locally advanced head and neck cancer: an RTOG analysis. J Clin Oncol 2008;26:3582-3589. 